\title{
Computational Developmental Neuroscience: Capturing Developmental Trajectories From Genes to Cognition
}

\author{
Jean-Philippe Thivierge
}

\begin{abstract}
Over the course of development, the central nervous system grows into a complex set of structures that ultimately controls our experiences and interactions with the world. To understand brain development, researchers must disentangle the contributions of genes, neural activity, synaptic plasticity, and intrinsic noise in guiding the growth of axons between brain regions. Here, we examine how computer simulations can shed light on neural development, making headway towards systems that self-organize into fully autonomous models of the brain. We argue that these simulations should focus on the "open-ended" nature of development, rather than a set of deterministic outcomes.
\end{abstract}

Index Terms-Axonal growth, computational model, development, intrinsic activity, synaptic plasticity.

\section{INTRODUCTION}

M OST of the neural circuits involved in cognition have developed an adult-like structure well before their involvement in sensation, perception, and behavior [1]. For instance, the topographic connectivity of primary sensory areas (visual, somatosensory, etc.) is already in place in the embryo, albeit lacking fine precision [2].

This ability of neurons to anticipate their final organization is realized by a highly specific process of growth and refinement of axonal projections that occurs independently of (and prior to) any visual experience. To understand this process, researchers across a variety of disciplines must account for several factors contributing to brain development. These factors include, but are not limited to, genetics, neural activity, synaptic plasticity, and intrinsic noise.

What are the prospects for an integrated, systems-level computational approach aimed at a mechanistic understanding of these factors and their interactions? To address this question, we begin by examining models of genetically driven development, then consider models that incorporate endogenous neural activity and synaptic plasticity. Building on insights from models of local neuronal circuits, we go on to discuss the formation of highly organized large-scale networks of anatomically distinct brain regions. Based on recent advances in the field, we argue

Manuscript received October 20, 2009; revised December 19, 2009. Date of publication January 29, 2010; date of current version March 12, 2010. The work was supported by a postdoctoral fellowship from NSERC (Canada) and FRSQ (Québec), as well as a grant from the National Academies Keck Futures Initiative.

The author is with the Department of Psychological and Brain Sciences, Indiana University, Bloomington, IN 47405 USA (e-mail: jthivier@indiana.edu).

Digital Object Identifier 10.1109/TAMD.2010.2041776 that the multiple intrinsic factors contributing to the wiring of neuronal connections, along with the dynamical nature of development, are within the grasp of an integrated computational approach. Such an approach will require proponents in the field of computational developmental neuroscience to adopt a developmental program that is biologically grounded, highly "open-ended," and stochastic. This approach will shed light on developmental processes, as well as their links to evolution, cognition, and behavior in the adult brain. Here, our goal is not to provide an exhaustive list of models, but rather to illustrate key principles of computational approaches by describing a few models at different levels of analysis, from single synapses all the way up to the large-scale cytoarchitecture of the brain.

\section{ACTIVITY-INDEPENDENT DEVELOPMENT-A BLUEPRINT FOR THE BRAIN?}

During an early stage of development, axonal elongations "sniff out" their correct anatomical position by following molecular guidance cues [3]. Several of these cues are expressed along gradients of concentration across various regions (e.g., a low-tohigh concentration from ventral to dorsal retina; see Fig. 1). In retinotectal projections (from retina to midbrain), for instance, genetic markers are expressed along both the sending region (retina) and the target region (superior colliculus or tectum) [4]. Axonal elongations from retinal ganglion cells use these molecular signals to establish synaptic contacts in termination zones of the target region such that point-to-point topography is maintained (in an inverse manner). This mechanism is not unique to the visual system; similar genetic markers are responsible for the layout of a rough topographic map in all primary sensory areas of cortex [2].

Computer models of neural development, with a tradition dating back several decades, have been instrumental in investigating the role of genes in shaping synaptic connectivity between brain regions. These models are not part of so-called "genetic algorithms," where a certain fitness function is known in advance. Here, more often than not, the fitness function of genetic markers is not assumed in advance.

One of the early theoretical constructs aimed at characterizing genetically guided connectivity is the chemoaffinity hypothesis [5]. A postulate of this viewpoint is that molecular labels expressed across sending and target regions must specify topographic maps. Following this proposal, several models have shown that molecular gradients account for a large body of experimental findings on normal and aberrant map formation (for a review, see [6]). 


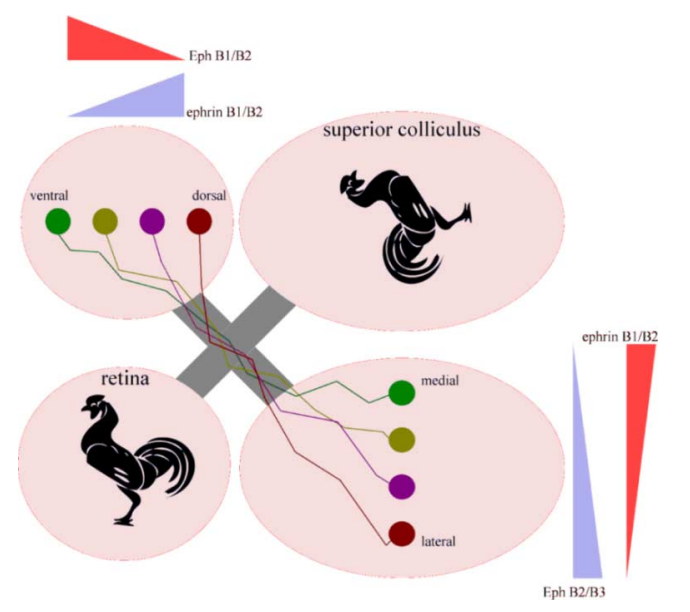

Fig. 1. Molecular guidance cues are responsible for the establishment of a rough topographic map between the retina and superior colliculus (SC, in the midbrain). An object on the retina (depicted as a rooster) is reproduced in the midbrain. Axons (colored lines) transmit information from the retina to the SC (initial and corresponding termination zones shown by filled circles). Only a single axis is shown here. Examples of gradients of expression for complementary molecular guidance cues (Eph/ephrin, [4]) are shown in triangles. These gradients provide information relevant to the migration of axons to correct topographic positions.

Most of these models, however, leave largely unanswered the question of whether gene expression follows particular optimization principles for guiding the growth of axonal projections. Several hypotheses about the nature of these optimization principles have been proposed. For instance, developmental processes may aim to minimize the wiring length necessary to link two regions of the brain [7]. Few models have attempted to relate optimization principles to the growth of axons, and those that have done so have resulted in conflicting accounts. Some models claim that growing axons make optimal decisions on growth direction in the presence of noise in molecular gradients [8], [9], while others claim that these growth decisions attempt to minimize the number of steps necessary to reach a target site [10].

One recent model, for instance, assumes that the path chosen by migrating axons can be described by a Bayesian "ideal observer" whose goal is to extract directional information from molecular gradients [9]. In this model, the growth cone (i.e., the tip of the axonal elongation that is sensitive to changes in molecular concentration) is attempting to determine whether the gradient is increasing or decreasing, given solely information on its receptor occupancy (Fig. 2). This is achieved by calculating the probability $P(\mu \mid \beta)$ of sensing a relative change in gradient concentration $(\mu)$ given receptor bindings $\beta$

$$
P(\mu \mid \beta) \propto P(\mu) \exp \left[(1-\hat{\rho}) \mu R_{b}\right]
$$

given

$$
\hat{\rho}=\int_{0}^{\infty} d \gamma P(\gamma) \frac{\gamma^{n}}{(1+\gamma)^{N}} \rho
$$

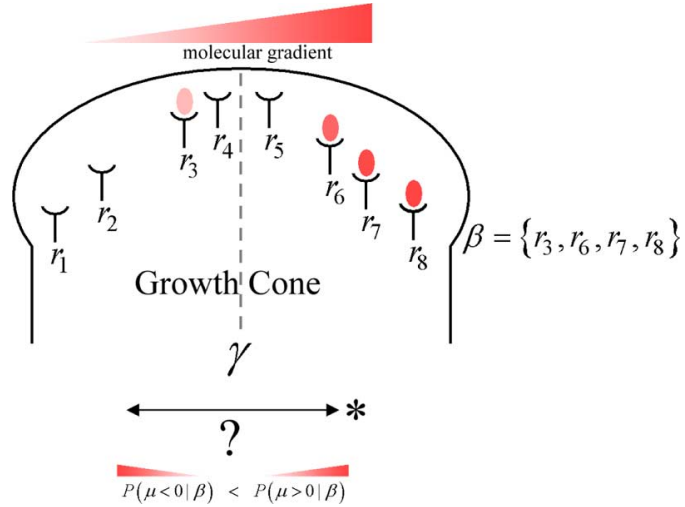

Fig. 2. A Bayesian model of growth cone migration [9]. Receptors are located at different positions $\left(r_{1}, \ldots, r_{8}\right)$ on the growth cone. The concentration of a molecular gradient is shown above the cone; $\gamma$ is the concentration at the center of the gradient sensor; $\mu$ is the relative change in gradient concentration across the sensing surface. Only a fraction of receptors are bound; these are shown by colored ellipses and form the set $\beta$. In the model, a relative value is assigned to the probability that $\mu$ is a decreasing or increasing gradient, given the receptor occupancy. In the example illustrated, the growth cone would move towards the right, as indicated by the asterisk ("*”).

and

$$
R_{b}=\sum_{r \in \beta} r
$$

where $N$ is the total number of receptors and $r$ is a receptor position. Equation (1) is based on the probability $\rho=\gamma /(1+\gamma)$ that a receptor is bound, where $\gamma$ is the gradient concentration at the center of the gradient sensor. By estimating $P(\mu \mid \beta)$ using (1), it can be shown that the growth cone maximizes its chances of correctly estimating the gradient direction.

Of course, caution is warranted in interpreting models based on principles of optimality, because alone they do not constitute an explicit means of implementing an optimal strategy for axonal growth.

Leaving aside debates about the optimal nature of axonal growth, the prevalence of genetically driven circuit formation makes it difficult to deny its role in the development of cognitively relevant circuits. More controversial, however, are the manifestations of that role. Many models of learning focus on the statistical extraction of information from structured environments, and fail to account for how experience-independent (i.e., endogenously driven) processes bias knowledge acquisition mechanisms in the first place [2]. There are many examples of ways in which gene expression influences the representation of knowledge. For instance, recent functional magnetic resonance imaging (fMRI) findings suggest that visual topography is preserved in higher regions of associative cortex that have traditionally been described as receiving a massive convergence of afferences from lower cortical areas [11]. The presence of topography in frontal cortex suggests that spatially organized information may be preserved beyond primary cortical layers. Given the known expression of molecular guidance cues in cortex [12], it is likely that these cues provide an early contribution to the organization and functionality of higher-order cortical circuits. 


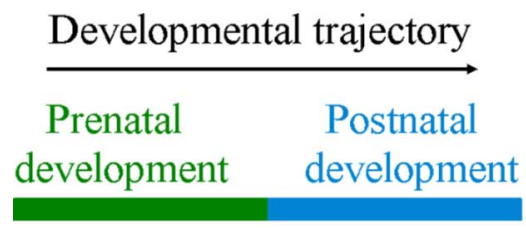

Molecular guidance

of axonal projections

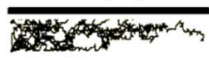

Spontaneous waves

of electrical activity

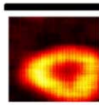

Experience-dependen modification of neural circuits

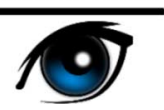

Fig. 3. The establishment of visual projections from retina to midbrain involves a series of processes that unfold over the course of development. In an early stage of prenatal development, axonal elongations are guided by molecular guidance cues. Subsequently, when synaptic contacts are established, highly correlated patterns of retinal activity emerge and propagate to the superior colliculus. Finally, during postnatal development, experience-dependent map formation is responsible for the refinement of connectivity.

Taken as a whole, the body of work on computational models of genetically driven brain development offers many potential avenues for devising developmental programs that bias particular forms of neural wirings and, ultimately, both sensory and cognitive processes. Molecular guidance, however, cannot tell the whole story on brain development, as it is limited to a welldefined period in vertebrate development (Fig. 3). Furthermore, the axonal projections resulting from molecular cues are typically imprecise in terms of their anatomical specificity. These considerations make it unlikely that molecular markers provide a deterministic blueprint for brain development.

\section{WHEN ACTIVITY-DEPENDENT DEVELOPMENT IS MAKING WAVES}

A large corpus of experimental work shows that electrical activity in the developing neural system contributes to the refinement of connectivity. The rate at which neurons produce action potentials, for instance, is related to their dendritic extent [13]. The facilitatory effect of activity in the early neural system is captured by computer models of neurite outgrowth [14]. Neural activity, however, has more than a simple facilitatory (all-or-none) effect on neural wiring. The patterns of spontaneous activity produced during development of the retina, for instance, are highly correlated in space and in time, providing a rich signal that can be used to drive map formation [15].

\section{A. Models of Endogenous Neural Activity}

In recent years, computational models have successfully captured the "wave-like" nature of spontaneous activity [16]-[19]. In particular, mean-field models (abstracting away details of single action potentials) can be designed to generate bursts of activity that propagate across the retina and produce highly correlated patterns among neighboring ganglion cells.

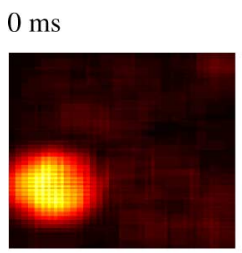

$200 \mathrm{~ms}$

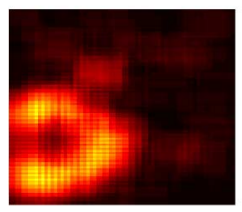

$100 \mathrm{~ms}$

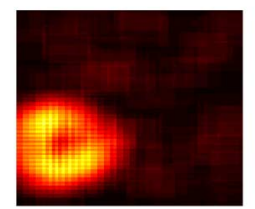

$300 \mathrm{~ms}$

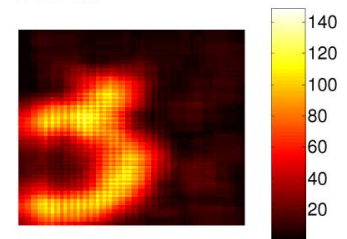

Fig. 4. Spontaneous wave-like activity in a model of retinal cells. Different frames show $300 \mathrm{~ms}$ of activity for a 2-D sheet of neurons. Color maps show the activation of retinal cells obtained from (4). For further details, see [34].

One model simulates the membrane potentials of amacrine and retinal ganglion cells in order to produce wave-like dynamics

$$
\begin{aligned}
X_{i}^{\text {retina }}(t) & =X_{i}^{\text {retina }}(t-1) \cdot \exp \left(-\Delta t / \tau_{\text {retina }}\right)+N_{\mathrm{AC}} \\
X_{i}^{\mathrm{AC}}(t) & =X_{i}^{\mathrm{AC}}(t-1) \cdot \exp \left(-\Delta t / \tau_{\mathrm{AC}}\right)+N_{\mathrm{AC}}
\end{aligned}
$$

where $t$ is a time-step of update; $i$ indexes different cells of the retinal (i.e., retinal ganglion cells) and amacrine populations; $\Delta t$ is the time-step of the model; $N_{\mathrm{AC}}$ corresponds to the number of amacrine cells that fired at the previous time-step $(t-1)$ within the spatial region $\phi_{\mathrm{AC}} ; \tau_{\text {retina }}$ and $\tau_{\mathrm{AC}}$ are the integration times of retinal and amacrine cells, respectively. In the model, amacrine cells receive their input from each other, and send their output to ganglion cells; the latter do not receive input from each other, and do not send activity back to the amacrine cells. At any given time-step, cells can fire spontaneously with a random probability (set to $p=0.03$ in order to obtain low firing rates). In addition, cells fire when either their membrane potential rises above threshold $\left(\theta_{\mathrm{AC}}\right)$ or if they are within an UP state (lasting for a predefined period of time). Further details and simulation results are available elsewhere [18], [34]; for an example of retinal wave-like activation, see Fig. 4.

A key aspect of the above model is that stochasticity is required to initiate waves at random spatial locations and with a random direction of propagation. Because spontaneous neural activity of a highly stochastic nature has been reported throughout the central nervous system in a variety of vertebrate species [20], it likely plays a ubiquitous role in development. Moreover, this role likely extends into adulthood, where intrinsic activity modulates sensory and behavioral processes [21], [22].

In order to understand how spontaneous activity inscribes itself in the developmental program of the brain, several conceptual gaps must be filled. Advances in computational models should aim to capture not just the influence of spontaneous activity at a particular period of development, but, more broadly, its trajectory from early to late stages of neural growth-a goal that is in line with the concept of a developmental program that spans the whole course of brain formation. 
Let us illustrate one of the challenges in modeling spontaneous activity from the vantage point of its developmental trajectory. In the forebrain, inhibitory interneurons are the first to develop functional synapses [23]. Initially, the neurotransmitter GABA functions in an excitatory fashion and drives spontaneous activity. The establishment of excitatory neurotransmission then switches GABA from excitatory to inhibitory. This peculiar aspect of development may explain why spontaneous waves of activity are limited to a brief period of development preceding eye opening [24]. Computational models that autonomously transition between stages of development would shed light on this phenomenon. A key question that these models must address is that of commitment- to what extent are developmental "decisions" at one stage subject to change during a subsequent stage? While complete determinism of early developmental stages would be implausible, some degree of commitment likely prevents rewiring of an initial map laid out by genes [25].

\section{B. Interplay Between Spontaneous Activity and Synaptic Plasticity}

Equally important is the question of how spontaneous activity interacts with mechanisms of synaptic plasticity to drive map formation. Because the influence of spontaneous waves on map formation is restricted to a brief time period (roughly 4-6 days), there is some developmental pressure to efficiently extract information from these waves and use it to refine axonal projections. As a consequence, the nervous system is likely to favor mechanisms of synaptic plasticity that quickly and accurately lead to a correct connectivity. While the role of synaptic plasticity as a major driving force in neural development is well established [26], how synaptic plasticity fulfils that role is a topic of sustained interest. The most likely candidates for synaptic plasticity are rules that detect correlated activity — most prominently, versions of Hebbian learning [27]. Models that incorporate such a principle can successfully account for map formation in the early visual system [28]. In essence, the mechanism at play is simple: neurons that "fire together" on the retina will tend to "wire together" in the midbrain. A variety of models have combined this mechanism with some form of competition for establishing postsynaptic contacts [29].

Taken together, these models suggest that a number of different possible rules, from spike-timing-dependent plasticity (STDP) to more coarse-grained rules based on mean firing rates (Fig. 5), can account for some aspects of map formation. This leaves unanswered the question of which specific rule is most plausible.

On the one hand, proponents of spike-based models argue that STDP is a well-documented rule for plasticity in the early visual system [30]-[32]. In addition, simulations show that it can successfully refine a rough topographic map and produce precisely organized projections [33], [34].

A phenomenological version of STDP relating changes in synaptic efficacy $\left(\Delta w_{i j}\right)$ to spike timing at a particular timepoint $t$ can be described as follows

$$
\Delta w_{i j}(t)= \begin{cases}W_{+} \cdot \exp \left(-\Delta t_{i j} / \tau_{+}\right) & \text {if } \Delta t_{i j}>0 \\ -W_{-} \cdot \exp \left(\Delta t_{i j} / \tau_{-}\right) & \text {if } \Delta t_{i j} \leq 0\end{cases}
$$
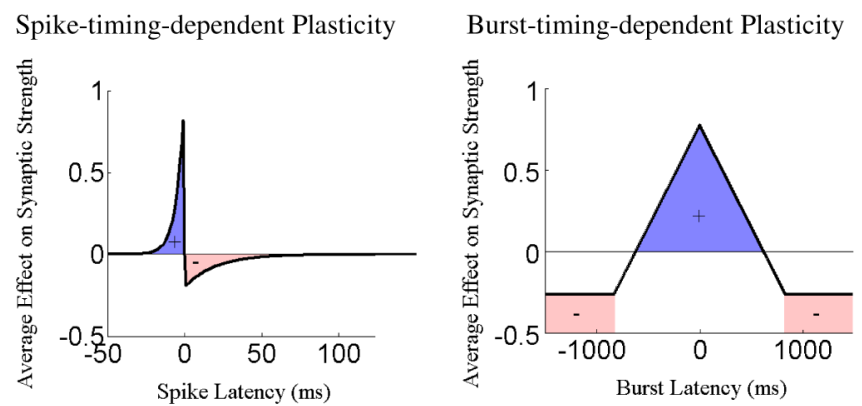

Fig. 5. Different rules for synaptic plasticity rely either on the STDP or on the latency between bursts (burst-timing-dependent plasticity), where each burst is composed of multiple spikes. Both plots relate spike/burst latency to changes in synaptic strength between two neurons. Blue: long-term potentiation (increase in strength); red: long-term depression (decrease in strength).

where $\Delta t_{i j}=t_{i}-t_{j}$ reflects the difference between the last spike arrival times of presynaptic $\left(t_{i}\right)$ and postsynaptic $\left(t_{j}\right)$ neurons; $W_{+}$and $W_{-}$control the magnitude of change in synaptic efficacy; $\tau_{+}$, and $\tau_{-}$control the time-course of plasticity.

On the other hand, spike-based rules are problematic because immature developing neurons are unreliable in propagating information at the level of single spikes, because they lack fine temporal resolution [35] and exhibit high levels of noise [36]. One alternative is a rule based on bursts of action potentials [37]. One version of this rule proposes a linear relationship between synaptic change and burst latency where a burst is composed of multiple spikes (Fig. 5). In recordings of the developing lateral geniculate nucleus, this burst-based rule captured a higher proportion of spikes than a spike-based rule, even when the latter rule was modified in a number of ways.

An intriguing possibility is that different rules for synaptic plasticity contribute to specific developmental stages. While an early stage may recruit a burst-based rule to extract information from spontaneous waves, a later stage may recruit a spike-based rule to extract information from the environment. A burst-based rule may be well-suited to spontaneous waves, which lead to much more correlated activity than natural visual environments. Future computational work examining developmental trajectories could address this question and propose rules for a plausible developmental program.

\section{Models of LARGe-SCALE BRAIN DeVElopMent}

The need to develop a biologically informed developmental program is further illustrated by zooming out of the individual synapse and examining development at the scale of broad anatomical regions. At this larger scale of neural connectivity, we find principles of development and organization that hold across mammalian species.

A prominent example of one such principle is the "inside-out" pattern of circuit formation. The development of local circuit properties occurs during a stage when different brain regions are functionally isolated from each other. The later growth of inter-region connectivity links these specialized modules into integrated neuronal networks. One example of the inside-out principle is the development of thalamocortical projections, where thalamic nuclei are functionally segregated prior to the establishment of synaptic contacts within specific cortical areas 
[38]. A local-to-distributed development, characteristic of the inside-out principle, is also reported in human resting-state fMRI [39], suggesting that this principle may subserve both anatomical and functional aspects of brain development.

At the functional level, an inside-out course of development contributes to the establishment of anatomically defined clusters with distinct functional roles, including visual, auditory, somatosensory-motor, and frontolimbic clusters in the mammalian brain [40]. The integration of information from these distinct clusters occurs through long-range projections and relay centers such as the thalamus. One crucial consequence of an inside-out development is that small changes in the local circuit properties of individual clusters can have a large impact on information processing once these clusters become interconnected.

Computational models are well-suited to test the consequences of local circuit properties on global neuronal processing. Importantly, these models can examine the link between developmental trajectories based on the inside-out principle and cognitive outcomes. Already several models of large-scale brain development have been proposed. These models capture several aspects of global connectivity, including a small-world organization [41], [42], dependence on spatial distance [43], economy of wiring length [44], and economy of processing steps [45]. In sum, these models suggest that several competing requisites shape neuronal connectivity during development.

To illustrate this idea, let us consider a relatively simple model of motor cortex organization [46] (Fig. 6). The goal of this model was to account for several mapping requisites, including a somatotopic map of the body, a map of hand location in space, and a partitioning of cortex into regions that emphasize different complex, ethologically relevant movements. A Kohonen network [47] was employed to balance out these conflicting requisites. This network was composed of a series of nodes laid out topographically. During training, these nodes received a set of inputs and learned to represent these inputs in a structured manner. After training, each node captured a subset of the inputs, with nodes that are close together sharing similar representations.

More formally, stimuli $\mathbf{v}$ from a stimulus space $V$ are mapped onto neurons located on the vertices $r$ of a grid in an output space $A$. Each neuron has a center field $\mathbf{w}_{\mathbf{r}}(\in V)$. The stimulus $\mathbf{v}$ is mapped in a winner-take-all fashion onto the neuron $\mathbf{r}$ that has a field center $\mathbf{w}_{\mathbf{r}}$ closest to $\mathbf{v}$

$$
\mathbf{v} \rightarrow \mathbf{r}:\left\|\mathbf{w}_{\mathbf{r}}-\mathbf{v}\right\|=\min _{\mathbf{r}^{\prime} \in A}\left\|\mathbf{w}_{\mathbf{r}^{\prime}-\mathbf{v}}\right\| .
$$

The map is learned by successive presentation of stimuli $\mathbf{v}$, and by shifting the field center of the winning neuron $\mathbf{r}$, as well as those of its neighbors $\mathbf{r}^{\prime}$ towards the stimuli

$$
\delta \mathbf{w}_{\mathbf{r}^{\prime}}=\in h_{\mathbf{r}, \mathbf{r}^{\prime}}\left(\mathbf{v}-\mathbf{w}_{\mathbf{r}}\right) .
$$

The neighborhood function $h_{\mathbf{r}, \mathbf{r}^{\prime}}$ takes a Gaussian form and is characterized by a length scale $\sigma$

$$
h_{\mathbf{r}, \mathbf{r}^{\prime}}=\exp -\frac{\left(\mathbf{r}-\mathbf{r}^{\prime}\right)^{2}}{2 \sigma^{2}}
$$
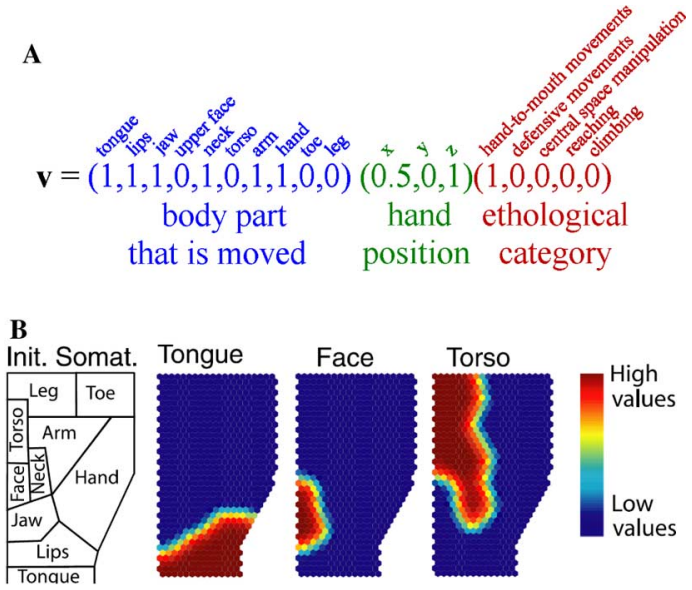

C
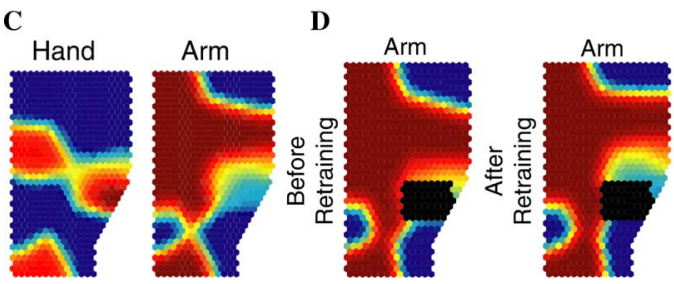

Fig. 6. Motor cortex organization is the result of a competition among map requisites. A. Design of the input vectors for the Kohonen network described in main text. Each input vector is comprised of three parts describing the body parts that are moved, the position of the hand, and the ethological category of the movement. The example shown represents a complex hand-to-mouth movement with activation of several limbs. B. Example of three body parts (tongue, face, and torso) that are mapped in a somatotopic fashion (left-most figure: complete body map used in the Kohonen network). C. Nonsomatotopic mapping of body parts involved in complex movements. D. Reorganization of the arm representation following lesion (black: site of lesion). B-D adapted from [46].

A two-dimensional grid of nodes was employed to represent the organization of motor cortex. These nodes received input vectors that described simple, as well as more complex movements. For instance, a complex hand-to-mouth movement consisted in the activation of the tongue, lips, jaw, neck, arm, and hand. In the model, this was described by an input vector [Fig. 6(A)] with three distinct parts: 1) a ten-dimensional binary vector identifying the body parts that are moved; 2 ) continuous values for the position of the hand in 3D Cartesian space (centimeters relative to the mouth); and 3) binary values for the ethological category of the movement.

A total of 12800 input vectors were presented during training. These vectors included complex movements for an equal proportion of ethological categories. The input vectors also included simpler movements of the foot, leg, mouth, lips, jaw, tongue, as well as movements of the arm to a variety of locations in space.

After training, the result is an artificial motor cortex that contains many features seen in actual motor cortex. One such feature is that many individual body parts in the artificial motor cortex followed somatotopic coordinates [Fig. 6(B)]. In addition, as in actual motor cortex, body parts involved in complex ethological movements did not respect a strict topography [Fig. 6(C)]; this is because these body parts were often coactivated with several other limbs. In other words, the strong correlation between activation of different limbs during 
execution of complex movements gave rise to nontopographic representations.

The idea that mapping is use dependent and sensitive to the statistics of the training input is illustrated by simulating a lesion where part of the map no longer received activity [Fig. 6(D)]. This simulation led to a reorganization of the map whereby the region surrounding the lesion "took over" the activation of the ablated area.

While the above example is a clear instance of anatomical segregation based on particular functional roles, the origins of functional specialization in large-scale brain organization (e.g., across several regions of cortex) are far less clear. Although few models have examined this issue, two conflicting viewpoints have emerged. One view is that functional specialization results from the modular nature of environmental goals [48]. This is supported by the findings that an artificial network faced with a variety of tasks can learn to self-organize into distinct (specialized) clusters. When applied to biology, this explanation becomes problematic, as the nervous system develops modular specialization well before any sensory experience [38]. By the age of two, the brain is already strongly modular and exhibits patterns of correlated resting state activity (i.e., in the absence of a task or stimulus) that are highly similar to those of adults [49].

A more plausible scenario is that endogenous mechanisms - such as molecular guidance cues and spontaneous activity - drive the emergence of modular specialization. That is not to say that endogenous processes are the sole driving force, as functional modules are reshaped until maturation [39]. Rather, the propensity towards a modular development emerges from endogenous rules. In accord with this viewpoint, recent computational work shows that, under a minimal set of assumptions, large-scale brain development organizes itself spontaneously into distinct modules [50].

In turn, a modular organization may impose important constraints on the neural dynamics underlying cognition [51]. Recent computational models have successfully captured some aspects of the relationship between the large-scale organization of anatomical connections and resting-state activity [52], [53]. Models of autonomous development should build on these efforts to examine the mutual influence of connectivity and neural dynamics at different stages of maturation.

\section{The Role of Developmental TRAJECTORIES IN EVOLUTION}

It is likely that the characteristics of brain development described thus far, from the molecular guidance of individual axons all the way up to interactions of large regions, are constrained by natural selection. According to one theory, natural selection can influence the presence, anatomical localization, and size of "cortical fields" that form basic functional units in neocortex [54].

One way in which natural selection influences brain architecture is through the expression of genes at specific stages of development. While many aspects of cortical organization are conserved across mammals, the slight "tweaking" of gene expression at a particular developmental stage can have drastic effects on neural structure. For instance, knockout mice that lack the $\beta 2$ subunit of the nicotinic acetylcholine receptor have abnormal forms of spontaneous activity during a brief period of development, in turn affecting the activity-dependent refinement of retinocollicular projections [24].

By considering developmental trajectories, as proposed here, we may gain insights into the relation between evolution and development. The mechanisms by which development tweaks gene expression may offer a principled way to control phenotypic outcomes, with earlier gene alterations leading to large effects compared to later gene alterations. Developmental alterations in gene expression are, however, under strict evolutionary constraints, as large changes in gene expression may jeopardize viability.

In computational work, fitness functions have been used to evaluate the viability of neural systems over a wide range of parameters [55]. However, it is not yet clear how fitness functions relate to evolutionary pressures, including the specific action of genetic, environmental, and intrinsic factors. These factors could become part of the next generation of computational theories, allowing more direct links between development and evolution.

\section{TOWARDS AN OPEN-ENDED, STOCHASTIC DEVELOPMENTAL PROGRAM}

What avenues could lead to an integrated computational approach to autonomous development that incorporates biological principles of single neurons as well as large-scale systems? As a starting point, recent models of the adult brain incorporate anatomical constraints and capture broad features of large-scale brain dynamics [52], [56]. To recast these models in a developmental context, one could begin with an under-developed architecture and explore developmental programs that give rise to appropriate brain circuits. Crucially, such programs should incorporate genes (e.g., molecular gradients), stochastic neural activity, and synaptic plasticity. How these factors are incorporated will depend upon the particular choice of model. For instance, a large-scale model of spiking neurons with STDP [56] could straightforwardly incorporate waves of endogenous activity (4) in early development [34].

Several questions could be asked with such a model. What programs would give rise to an inside-out development of connectivity (see Section IV)? Would the development of functional connectivity mirror the growth of anatomical connections? How does the development of individual cortical regions affect more global aspects of information processing in the brain? What is the impact of different developmental trajectories on outcomes such as learning and memory in the mature brain?

Computational models could complement experimental data by providing a more complete account of development, unconstrained by the limitations of current technologies. For instance, fMRI can estimate patterns of brain activation, but lacks single-neuron resolution and cannot be employed in early stages of development. More invasive techniques, including two-photon imaging as well as the use of knockout models, allow only limited parallels with human development, in part because of incomplete homologies between humans and other mammals [57]. Computational models composed of large networks of artificial neurons could reach beyond experimental 
insights by simulating how genes and neural activity contribute to the growth of connectivity from the early stages of development.

When devising such models, one must be cognizant of potential pitfalls, including the over-interpretation of results obtained from abstract models as well as the design of overly complex models that lack testable predictions. Models must strike a balance between abstracting away neurobiological details of neural circuits, and generating precise hypotheses on the role of particular developmental events.

\section{CONCLUSION}

In the current work, we have emphasized that developmental programs in the brain are "open-ended," meaning that they are not fixed from the early stages of neural outgrowth. The noisy expression of molecular guidance cues, combined with the stochasticity of spontaneous neural activity, suggest that the developmental program of the brain is highly nondeterministic in nature. One advantage of an open-ended developmental trajectory is that processes at later stages can build upon earlier ones to refine axonal projections (as is the case with activity-dependent mechanisms) or to link together specialized modules with distinct yet complementary functional roles (as is the case with large-scale connectivity).

The success of computational approaches in capturing several aspects of neural development opens prospects for an integrated systems approach leading to embedded models of brain development. This approach will shed light on the causal rules linking genes, connectivity and cognition, serving as both an explanatory and predictive tool. Because of the open-ended nature of development, computational approaches must focus on processes - chains of events that drive development - and not just a set of deterministic (or seemingly optimal) outcomes.

\section{ACKNOWLEDGMENT}

The author is thankful to O. Sporns and E. Balaban for insightful discussions, as well as two anonymous reviewers for their useful comments.

\section{REFERENCES}

[1] E. Balaban, "Cognitive developmental biology: History, process and fortune's wheel," Cognition, vol. 101, pp. 298-332, 2006.

[2] J. P. Thivierge and G. F. Marcus, "The topographic brain: From neural connectivity to cognition," Trends Neurosci., vol. 30, pp. 251-259, 2007.

[3] A. Martinez and E. Soriano, "Functions of ephrin/Eph interactions in the development of the nervous system: Emphasis on the hippocampal system," Brain Res. Rev., vol. 49, pp. 211-226, 2005.

[4] T. McLaughlin and D. O'Leary, "Molecular gradients and development of retinotopic maps," Аnnu. Rev. Neurosci., vol. 28, pp. 327-355, 2005.

[5] R. W. Sperry, "Chemoaffinity in the orderly growth of nerve fiber patterns and connections," Proc. Nat. Acad. Sci. USA, vol. 50, pp. 703-710, 1963.

[6] G. Goodhill and J. Xu, "The development of retinotectal maps: A review of models based on molecular gradients," Netw.: Comput. Neural Sys., vol. 16, pp. 5-34, 2005.

[7] G. Buzsaki, C. Geisler, D. A. Henze, and X. J. Wang, "Interneuron diversity series: Circuit complexity and axon wiring economy of cortical interneurons," Trends Neurosci., vol. 27, pp. 186-193, 2004.
[8] B. W. Andrews and P. A. Iglesias, "An information-theoretic characterization of the optimal gradient sensing response of cells," PLoS Comput. Biol., vol. 3, p. e153, 2007.

[9] D. Mortimer, J. Feldner, T. Vaughan, I. Vetter, Z. Pujic, W. J. Rosoff, K. Burrage, P. Dayan, L. J. Richards, and G. J. Goodhill, "Bayesian model predicts the response of axons to molecular gradients," Proc. Nat. Acad. Sci. USA, vol. 106, pp. 10296-10301, 2009.

[10] J. P. Thivierge and E. Balaban, "Getting into shape: Optimal ligand gradients for axonal guidance," Biosystems, vol. 90, pp. 61-77, 2007.

[11] M. A. Silver and S. Kastner, "Topographic maps in human frontal and parietal cortex," Trends Cogn. Sci., in press.

[12] E. Stein, N. Savaskan, O. Ninnemann, R. Nitsch, R. Zhou, and T. Skutella, "A role for the Eph ligand ephrin-A3 in entorhino-hippocampal axon targeting," J. Neurosci., vol. 19, pp. 8885-8893, 1999.

[13] Z. F. Mainen and T. J. Sejnowski, "Influence of dendritic structure on firing pattern in model neocortical neurons," Nature, vol. 382, pp. 363-366, 1996.

[14] C. van Oss and A. van Ooyen, "Effects of inhibition on neural network development through activity-dependent neurite outgrowth," J. Theor. Biol., vol. 185, pp. 263-280, 1997.

[15] E. Ruthazer, C. Akerman, and H. Cline, "Control of axon branch dynamics by correlated activity in vivo," Science, vol. 301, pp. 66-70, 2003.

[16] D. A. Butts, M. Feller, C. Shatz, and D. Rokhsar, "Retinal waves are governed by collective network properties," J. Neurosci., vol. 19, pp. 3580-3593, 1999.

[17] T. Elliott and N. Shadbolt, "A neurotrophic model of the development of the retinogeniculocortical pathway induced by spontaneous retinal waves," J. Neurosci., vol. 19, pp. 7951-7970, 1999.

[18] M. Feller, D. A. Butts, H. Aaron, D. Rokhsar, and C. Shatz, "Dynamic processes shape spatiotemporal properties of retinal waves," Neuron, vol. 19, pp. 293-306, 1997.

[19] M. H. Hennig, C. Adams, D. Willshaw, and E. Sernagor, "Early-stage waves in the retinal network emerge close to a critical state transition between local and global functional connectivity," J. Neurosci., vol. 29, pp. 1077-1086, 2009.

[20] M. Feller, "Spontaneous correlated activity in developing neural circuits," Neuron, vol. 22, pp. 653-656, 1999.

[21] A. Sapir, G. d'Avossa, M. McAvoy, G. L. Shulman, and M. Cor betta, "Brain signals for spatial attention predict performance in a motion discrimination task," Proc. Nat. Acad. Sci. USA, vol. 102, pp. 17810-17815, 2005.

[22] M. D. Fox, A. Z. Snyder, J. L. Vincent, and M. E. Raichle, "Intrinsic fluctuations within cortical systems account for intertrial variability in human behavior," Neuron, vol. 56, pp. 171-184, 2007.

[23] Y. Ben-Ari, "Developing networks play a similar melody," Trends Neurosci., vol. 24, pp. 353-360, 2001.

[24] T. McLaughlin, C. Torborg, M. Feller, and D. D. M. O'Leary, "Retinotopic map refinement requires spontaneous retinal waves during a brief critical period of development," Neuron, vol. 40, pp. 1147-1160, 2003.

[25] A. Chandrasekaran, D. Plaas, E. Gonzalez, and M. Crair, "Evidence for an instructive role of retinal activity in retinotopic map refinement in the superior colliculus of the mouse," J. Neurosci, vol. 25, pp. 6929-6938, 2005.

[26] T. H. Brown, E. W. Kairiss, and C. L. Keenan, "Hebbian synapses: Biophysical mechanisms and algorithms," Annu. Rev. Neurosci., vol. 13, pp. 475-511, 1990.

[27] D. O. Hebb, The Organization of Behavior. New York: Wiley, 1949.

[28] D. Tsigankov and A. Koulakov, "A unifying model for activity-dependent and activity-independent mechanisms predicts complete structure of topographic maps in ephrin-A deficient mice," J. Comput. Neurosci., vol. 21, pp. 101-114, 2006.

[29] L. Benuskova, F. F. Ebner, M. E. Diamond, and M. Armstrong-James, "Computational study of experience-dependent plasticity in adult rat cortical barrel-column," Network, vol. 10, pp. 303-323, 1999.

[30] K. Fox and R. Wong, "A comparison of experience-dependent plasticity in the visual and somatosensory systems," Neuron, vol. 48, pp. 465-477, 2005

[31] Y. Mu and M. M. Poo, "Spike timing-dependent LTP/LTD mediates visual experience-dependent plasticity in a developing retinotectal system," Neuron, vol. 50, pp. 115-125, 2006.

[32] L. Zhang, H. Tao, C. Holt, W. Harris, and M. M. Poo, "A critical window for cooperation and competition among developing retinotectal synapses," Nature, vol. 395, pp. 37-44, 1998.

[33] S. Song and L. F. Abbott, "Cortical development and remapping through spike timing-dependent plasticity," Neuron, vol. 32, pp. 339-350, 2001. 
[34] J. P. Thivierge, "How does non-random spontaneous activity contribute to brain development?," Neural. Netw., vol. 22, pp. 901-912, 2009.

[35] A. S. Ramoa and D. A. McCormick, "Enhanced activation of NMDA receptor responses at the immature retinogeniculate synapse," $\mathrm{J}$. Neurosci., vol. 14, pp. 2098-2105, 1994.

[36] J. E. Lisman, "Bursts as a unit of neural information: Making unreliable synapses reliable," Trends Neurosci, vol. 20, pp. 38-43, 1997.

[37] D. A. Butts, P. O. Kanold, and C. J. Shatz, "A burst-based "Hebbian" learning rule at retinogeniculate synapses links retinal waves to activity-dependent refinement," PLoS Biol., vol. 5, p. e61, 2007.

[38] P. Vanderhaeghen and F. Polleux, "Developmental mechanisms patterning thalamocortical projections: Intrinsic, extrinsic and in between," Trends Neurosci., vol. 27, pp. 384-391, 2004.

[39] D. A. Fair, A. L. Cohen, J. D. Power, N. U. Dosenbach, J. A. Church, F. M. Miezin, B. L. Schlaggar, and S. E. Petersen, "Functional brain networks develop from a "local to distributed" organization," PLoS Comput. Biol., vol. 5, p. e1000381, 2009.

[40] C. C. Hilgetag, G. A. Burns, M. A. O'Neill, J. W. Scannell, and M. P. Young, "Anatomical connectivity defines the organization of clusters of cortical areas in the macaque monkey and the cat," Philos. Trans. Roy. Soc. London B, Biol. Sci., vol. 355, pp. 91-110, 2000.

[41] M. Rubinov, A. R. McIntosh, M. J. Valenzuela, and M. Breakspear, "Simulation of neuronal death and network recovery in a computational model of distributed cortical activity," Amer. J. Geriatr. Psychiat., vol. 17, pp. 210-217, 2009.

[42] C. W. Shin and S. Kim, "Self-organized criticality and scale-free properties in emergent functional neural networks," Phys. Rev. E, Stat. Nonlin. Soft Matter. Phys., vol. 74, p. 045101, 2006.

[43] M. Kaiser and C. C. Hilgetag, "Spatial growth of real-world networks," Phys. Rev. E, Stat. Nonlin. Soft Matter. Phys., vol. 69, p. 036103, 2004.

[44] C. Cherniak, Z. Mokhtarzada, R. Rodriguez-Esteban, and K. Changizi, "Global optimization of cerebral cortex layout," Proc. Nat. Acad. Sci. USA, vol. 101, pp. 1081-1086, 2004.

[45] M. Kaiser and C. C. Hilgetag, "Nonoptimal component placement, but short processing paths, due to long-distance projections in neural systems," PLoS Comput. Biol., vol. 2, p. e95, 2006.

[46] T. N. Aflalo and M. S. Graziano, "Possible origins of the complex topographic organization of motor cortex: Reduction of a multidimensional space onto a two-dimensional array," J. Neurosci., vol. 26, pp. 6288-6297, 2006.

[47] T. Kohonen, Self-Organizing Maps. London: Springer-Verlag, 2001.

[48] N. Kashtan and U. Alon, "Spontaneous evolution of modularity and network motifs," Proc. Nat. Acad. Sci. USA, vol. 102, pp. 13773-13778, 2005.
[49] W. Gao, H. Zhu, K. S. Giovanello, J. K. Smith, D. Shen, J. H. Gilmore, and W. Lin, "Evidence on the emergence of the brain's default network from 2-week-old to 2-year-old healthy pediatric subjects," Proc. Nat. Acad. Sci. USA, vol. 106, pp. 6790-6795, 2009.

[50] M. Kaiser and C. C. Hilgetag, "Development of multi-cluster cortical networks by time windows for spatial growth," Neurocomputing, vol. 70, pp. 1829-1832, 2007.

[51] S. L. Bressler, "Large-scale cortical networks and cognition," Brain Res. Brain. Res. Rev., vol. 20, pp. 288-304, 1995.

[52] C. J. Honey, R. Kotter, M. Breakspear, and O. Sporns, "Network structure of cerebral cortex shapes functional connectivity on multiple time scales," Proc. Nat. Acad. Sci. USA, vol. 104, pp. 10240-10245, 2007.

[53] M. Muller-Linow, C. C. Hilgetag, and M. T. Hutt, "Organization of excitable dynamics in hierarchical biological networks," PLoS Comput. Biol., vol. 4, p. e1000190, 2008.

[54] L. Krubitzer, "The magnificent compromise: Cortical field evolution in mammals," Neuron, vol. 56, pp. 201-208, 2007.

[55] P. Achard and E. De Schtter, "Complex parameter landscape for a complex neuron model," PLoS Comput. Biol., vol. 4, p. e94, 2006.

[56] E. M. Izhikevich and G. M. Edelman, "Large-scale model of mammalian thalamocortical systems," Proc. Nat. Acad. Sci. USA, vol. 105, pp. 3593-3598, 2008.

[57] M. I. Sereno and R. B. H. Tootell, "From monkeys to humans: What do we now know about brain homologies?," Curr. Op. Neurobiol., vol. 15, pp. 135-144, 2005.

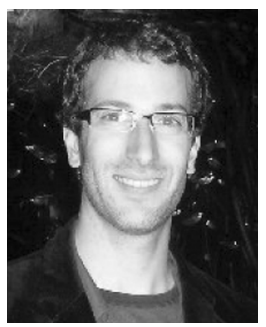

Jean-Philippe Thivierge received the B.A. degree in psychology from the University of Ottawa, Ontario, Canada, in 2001, the Ph.D. degree in psychology from McGill University, Montreal, Canada, in 2006.

$\mathrm{He}$ is currently an Assistant Scientist in the Department of Psychological and Brain Sciences, Indiana University, Bloomington. His research focuses on computational models of neural development, with an emphasis on the role of genes, spontaneous activity, and synaptic plasticity in the formation of circuits relevant to sensory and cognitive processes. He has authored or coauthored over 30 journal publications and conference proceedings.

$\mathrm{He}$ is actively involved in the organization of the IEEE International Joint Conference on Neural Networks, where he has acted as local chair, workshop chair, and technical committee cochair. 